\title{
Antenna-assisted picosecond control of nanoscale phase transition in vanadium dioxide
}

\author{
Otto L Muskens ${ }^{1}$, Luca Bergamini ${ }^{2,3}$, Yudong Wang ${ }^{1,4}$, Jeffrey M Gaskell ${ }^{5}$, Nerea Zabala ${ }^{2,3}$, CH de Groot ${ }^{4}$, \\ David W Sheel ${ }^{5}$ and Javier Aizpurua ${ }^{3}$
}

Nanoscale devices in which the interaction with light can be configured using external control signals hold great interest for next-generation optoelectronic circuits. Materials exhibiting a structural or electronic phase transition offer a large modulation contrast with multi-level optical switching and memory functionalities. In addition, plasmonic nanoantennas can provide an efficient enhancement mechanism for both the optically induced excitation and the readout of materials strategically positioned in their local environment. Here, we demonstrate picosecond all-optical switching of the local phase transition in plasmonic antenna-vanadium dioxide $\left(\mathrm{VO}_{2}\right)$ hybrids, exploiting strong resonant field enhancement and selective optical pumping in plasmonic hotspots. Polarization- and wavelength-dependent pump-probe spectroscopy of multifrequency crossed antenna arrays shows that nanoscale optical switching in plasmonic hotspots does not affect neighboring antennas placed within $100 \mathrm{~nm}$ of the excited antennas. The antenna-assisted pumping mechanism is confirmed by numerical model calculations of the resonant, antenna-mediated local heating on a picosecond time scale. The hybrid, nanoscale excitation mechanism results in 20 times reduced switching energies and 5 times faster recovery times than a $\mathrm{VO}_{2}$ film without antennas, enabling fully reversible switching at over two million cycles per second and at local switching energies in the picojoule range. The hybrid solution of antennas and $\mathrm{VO}_{2}$ provides a conceptual framework to merge the field localization and phase-transition response, enabling precise, nanoscale optical memory functionalities.

Light: Science \& Applications (2016) 5, e16173; doi:10.1038/Isa.2016.173; published online 21 October 2016

Keywords: insulator-metal phase transition; nanoantenna; plasmonics; $\mathrm{VO}_{2}$

\section{INTRODUCTION}

With the miniaturization of optical components and the convergence of electronic and photonic technologies, ultracompact devices are needed to control and switch light on length scales comparable to the optical wavelength. Resonant plasmonic antennas at visible and nearinfrared wavelengths offer the capability to concentrate energy in space and time and enhance light-matter interaction and nonlinear response ${ }^{1,2}$. To achieve order-unity switching effects, hybrid devices combining plasmonic antennas with materials showing a structural or electronic phase transition provide unique opportunities because they can provide very large changes in optical response. Recent investigations of such hybrid systems include switchable metal hydrides ${ }^{3-5}$, interface-mediated solid-liquid transitions of gallium ${ }^{6}$, resonant bonding phase-change materials $s^{7-10}$ and insulator-to-metal transitions (IMTs) in correlated oxides ${ }^{11}$. Chalcogenide phase-change materials, such as Ge:Sb:Te (GST), allow for non-volatile writing and rewriting of photonic devices using a pulsed laser ${ }^{8-10,12,13}$. Excitation of this phase-change response using the optical near-field opens up routes for achieving ultrasmall switching volumes and low-energy devices ${ }^{14,15}$.
Compared with chalcogenide phase-change materials, which provide rewritable, non-volatile memory functionality at relatively high temperatures, vanadium dioxide $\left(\mathrm{VO}_{2}\right)$ is characterized by a reversible IMTs at only modestly elevated temperatures of $\sim 68^{\circ} \mathrm{C}^{11,16}$. This phase transition can be driven using ultrafast laser pulses, where the precise mechanisms depend on the pulse duration and energy injection mechanisms involved, with a structural bottleneck as short as $80 \mathrm{fs}^{17-19}$. While strong nonequilibrium physics occur on ultrafast time scales, the $\mathrm{VO}_{2}$ IMT is quasi-thermal for times $>1 \mathrm{ps}^{18}$.

Several studies have reported the effects of a global, thermally or optically driven phase transition in $\mathrm{VO}_{2}$ on the plasmonic response of nanoparticles and metamaterials over a broad spectral range from terahertz ${ }^{20-23}$, mid-infrared ${ }^{24,25}$, near-infrared ${ }^{26-28}$ to visible ${ }^{29,30}$. In the $\mathrm{THz}$ range, the giant electric-field strength in micrometer-sized antenna feed gaps has been used to overcome the Coulomb potential barrier for IMT, analogous to DC field-induced switching ${ }^{21}$. In the visible and near-infrared, studies of plasmonic effects on the optical excitation of the IMT have focused on hot-electron injection-driven ultrafast processes ${ }^{31,32}$ and on non-resonant, continuous-wavemediated plasmonic heating 28,33 .

${ }^{1}$ Physics and Astronomy, Faculty of Physical Sciences and Engineering, University of Southampton, Southampton SO17 1BJ, UK; ${ }^{2}$ Department of Electricity and Electronics, FCTZTF, UPV-EHU, Bilbao 48080, Spain; ${ }^{3}$ Materials Physics Center, CSIC-UPV/EHU and DIPC, San Sebastian 20018, Spain; ${ }^{4}$ Nano Group, Faculty of Physical Sciences and Engineering, University of Southampton, Southampton S017 1BJ, UK and ${ }^{5}$ Materials and Physics Research Centre, University of Salford, Manchester M5 4WT, UK Correspondence: OL Muskens, Email: O.Muskens@soton.ac.uk

Received 27 February 2016; revised 31 May 2016; accepted 2 June 2016; accepted article preview online 3 June 2016 
Here, we use picosecond pulsed excitation to drive a highly localized phase-transition around resonant plasmonic nanoantennas. The picosecond pulsed regime is of relevance for many practical applications, and nonlinear devices on this time scale are needed to enable alloptical switching ${ }^{34}$. Our experiments are enabled by very high-quality $\mathrm{VO}_{2}$ films that are grown with a surface roughness $<10 \mathrm{~nm}$ using atmospheric chemical vapor deposition ${ }^{35}$. Gold nanoantennas were fabricated on top of these films, forming a hybrid system together with the $\mathrm{VO}_{2}$, with an effective response that is governed by the nanoscale interplay between the plasmonic and IMT components. Resonant antenna-assisted switching is studied using optical pump-probe experiments, where we perform picosecond pulsed excitation at a fixed pump wavelength while tuning the antenna resonance condition via the antenna length. Multifrequency arrays of crossed antennas ${ }^{36}$ were specifically designed to individually address the cross-coupling of optically induced phase transitions between adjacent antennas with mutual separations $<100 \mathrm{~nm}$. Antenna-assisted optical pumping is identified through the dependence of the IMT switching on the polarizations of the pump and readout, on the pulse energy of the optical pump, and on the cycling rate and hysteresis of the IMT. A model combining resonant antenna-mediated absorption, heat diffusion and thermally induced local IMT successfully explains the resonant pumping on picosecond time scales. Nanoantenna- $\mathrm{VO}_{2}$ hybrids with fast, picosecond response and picojoule switching energies enable new directions in all-optical switching, active control of quantum emission ${ }^{37}$ and plasmonic memristor-type devices exploiting nanoscale thermal memory ${ }^{28,38}$.

\section{MATERIALS AND METHODS}

\section{Fabrication of $\mathrm{VO}_{2}$ thin films}

$\mathrm{VO}_{2}$ thin films with low surface roughness were fabricated using atmospheric pressure chemical vapor deposition following the methods described elsewhere ${ }^{35}$. In short, $\mathrm{VO}_{2}$ films were deposited on boroaluminosilicate glass (Eagle by Corning, St Davids Park, UK) coated with a 30-nm layer of fluorine-doped tin oxide (FTO). The inclusion of this intermediate layer allowed the production of $\mathrm{VO}_{2}$ films with low surface roughness and suitable thermochromic transition temperature. FTO films were deposited onto 1-mm boroaluminosilicate glass using a precursor mixture of monobutyltintrichloride (MBTC), oxygen, water and trifluoroacetic acid (TFA). A laboratory-scale atmospheric pressure chemical vapor deposition (CVD) coater was used. The substrate was heated to $600{ }^{\circ} \mathrm{C}$ and repeatedly translated underneath a coating head to produce a $30-\mathrm{nm}$ thick layer. MBTC was heated to $95^{\circ} \mathrm{C}$ in a stainless-steel bubbler and introduced to a CVD reactor in a nitrogen carrier gas flow. Water and TFA were mixed to give a $0.1 \mathrm{M}$ solution and introduced to the CVD reactor by flash evaporation in a nitrogen carrier gas flow.

$\mathrm{VO}_{2}$ was deposited on top of the FTO layer using a precursor mixture of vanadium chloride and water in a 'static' cold wall atmospheric pressure CVD reactor. The substrate was sealed in a reaction chamber, allowing the area to be pressure-purged and backfilled with nitrogen, producing a controlled, oxygen-free environment for deposition. The temperature was increased to the coating temperature, and the reactants were passed parallel over the substrate for $1 \mathrm{~min}$ to produce a 50-nm coating. Vanadium chloride was heated to $100^{\circ} \mathrm{C}$ in a stainless-steel bubbler and was introduced into the coater in a nitrogen carrier gas flow. Water was heated to $60^{\circ} \mathrm{C}$ in a stainless-steel bubbler and was introduced to the coater in a nitrogen carrier gas flow. After film deposition, the sample was cooled to $<200^{\circ} \mathrm{C}$ before removal from the coater to prevent oxidation of the coating.

\section{Fabrication of nanoantennas}

Gold nanoantennas were fabricated on top of the $50-\mathrm{nm} \mathrm{VO}_{2}$ layer using e-beam lithography (JBX-9300FS by JEOL, Tokyo, Japan) at $100 \mathrm{keV}$ with a dose of $650 \mu \mathrm{C} \mathrm{cm}^{-2}$. After development, $45 \mathrm{~nm}$ of gold was evaporated followed by lift off in acetone. The width of the antenna arms was fixed at $80 \mathrm{~nm}$, and the length was varied between 160 and $360 \mathrm{~nm}$. A Ti layer of 5-nm thickness was used to improve the adhesion of the gold to the $\mathrm{VO}_{2}$. Helium-ion microscopy (HIM) was performed on all arrays under study (Orion, Zeiss, Jena, Germany). Compared with conventional electron microscopy, the helium-ion microscope allows high-resolution imaging of non-conducting samples without charging the substrate.

\section{Optical experiments}

The dielectric function of the $\mathrm{VO}_{2}$ film was characterized using variable-angle ellipsometry (Uvisel 2, Horiba, Stanmore, UK) ${ }^{35}$. Antenna experiments were performed using a picosecond pulsed Yb-fiber laser (Fianium, Hamble, UK) with a variable repetition rate between 0.1 and $20 \mathrm{MHz}$ and a fixed pulse duration of $9.2 \pm 0.1 \mathrm{ps}$, as determined using an autocorrelator (FR-103XL, Femtochrome, Berkeley, CA, USA). The oscillator output was amplified using two separate amplifier stages, one of which was used as the pump laser, with a fixed wavelength of $1060 \mathrm{~nm}$, and the second output was used to produce a broadband supercontinuum spanning the range $400-2500 \mathrm{~nm}$. A spectral band with tuneable centre wavelength and $2 \%$ spectral width was selected using a double-prism monochromator in subtractive mode. The 1060-nm pump and the continuously tuneable probe were combined onto an 1100-nm long-pass dichroic beam splitter and were focused onto the array using a 0.5 numerical aperture (NA), reflective Cassegrain objective (Edmund Optics, York, UK). All reported energies were measured before the objective, which has a transmission of $82 \%$. Approximately $65 \%$ of the transmitted intensity was focused to a waist of $1.4 \pm 0.1 \mu \mathrm{m}$, while $35 \%$ of the intensity was contained in much weaker satellite structures of the Cassegrain point-spread function extending up to several micrometers from the focus. Fluence values were calculated for the central $6 \pm 1 \mu \mathrm{m}^{2}$ focus area taking into account these factors. The absolute transmission measurements and pump-probe spectroscopy were obtained using a combination of optical choppers and lock-in detection. A motorized variable optical density (OD) filter (OD 2.0) was used to scan the pump power between 1 and $100 \%$ of the available range, with $100 \%$ corresponding to $13 \mathrm{~nJ}$ of pulse energy. For experiments without optical pumping, extinction measurements over a larger spectral window from the visible were collected by removing the dichroic splitter. The sample was mounted on a heater stage with a temperature controller capable of maintaining stable temperatures in the range $25-80^{\circ} \mathrm{C}$.

\section{Numerical modeling}

The optical response of the antennas on the $\mathrm{VO}_{2}$-glass substrate stack was modeled using FDTD simulations (Lumerical, Vancouver, BC, Canada). Following detailed HIM images (see Supplementary Fig. S3), the antennas were modeled as semi-cylinders with rounded end caps. Three-dimensional near-field absorption profiles were extracted and used as the source distribution for a heat-transport simulation performed using the Multiphysics finite element software (COMSOL 5.1, Cambridge, UK). The resulting temperature profiles were used to generate a three-dimensional contour map of the phase-change response around the antenna, which was subsequently used to calculate the modified antenna resonances. A more extensive description of the theoretical model is given in the Supplementary Section II. 


\section{RESULTS AND DISCUSSION}

Thermally induced phase transition of antenna- $\mathrm{VO}_{2}$ hybrids

To investigate the resonant antenna-assisted pumping of the $\mathrm{VO}_{2}$ IMT, arrays of crossed gold nanoantennas were fabricated on top of a $\mathrm{VO}_{2}$ film, with antenna lengths varying from 160 to $360 \mathrm{~nm}$ in steps of $50 \mathrm{~nm}$ (HIM images shown in Supplementary Fig. S2). A continuous $\mathrm{VO}_{2}$ film of $50-\mathrm{nm}$ thickness with a roughness of $5 \mathrm{~nm}$, estimated using atomic force microscopy, was used as the substrate for depositing gold nanoantennas using e-beam lithography.

Figure 1a shows five selected arrays, where the vertical antenna length $L_{\mathrm{v}}$ was kept fixed at $160 \mathrm{~nm}$ while the length $L_{\mathrm{h}}$ of the horizontal antennas was varied. This subset represents the behavior of all arrays of antennas, which follow very similar trends (see Supplementary Fig. S5). Figure 1b presents horizontally polarized extinction spectra (OD) for temperatures below (black) and above (red) the critical phase transition. A background spectrum was subtracted from all spectra corresponding to the bare $\mathrm{VO}_{2}$ substrate (see Supplementary Fig. S1). At temperatures far below the phase transition, the spectra show pronounced optical resonances in the near-infrared corresponding to the longitudinal half-wave $(\lambda / 2)$ antenna mode with a resonance wavelength shifting from 950 to $1500 \mathrm{~nm}$ with increasing antenna length. Above the phase transition (red lines, $80^{\circ} \mathrm{C}$ ) these resonant modes are strongly suppressed. This suppression can be qualitatively understood by the large changes in the optical permittivity of the $\mathrm{VO}_{2}$ film ${ }^{35}$, which is characterized by a strong reduction of the real part and an increase of the imaginary part of the permittivity in the infrared. The $\mathrm{VO}_{2}$ film acts as a load on the antenna response, resulting in both a blueshift and strong damping of its resonance.

The spectral response at temperatures below and above the phase transition is reproduced well by numerical calculations of the antenna- $-\mathrm{VO}_{2}$ hybrid, as shown in Figure $1 \mathrm{~b}$, obtained using the measured dielectric function of the $\mathrm{VO}_{2}$ film, as detailed in the
Supplementary Section II. Good agreement is obtained for the shape and amplitude of the spectra both below and above the phase transition, showing that a suppression of the antenna resonance OD by up to $60 \%$ is achieved through temperature control of the $\mathrm{VO}_{2}$ substrate. A small adjustment of all antenna lengths to 20-nm lower values was made to improve the agreement of the spectral resonance positions at low temperature. Furthermore, for shorter antennas with resonances near the visible range, the calculated antenna resonances are somewhat broadened compared with the experimental data, indicating an overestimate of the local absorption in the $\mathrm{VO}_{2}$ substrate in this spectral range. We attribute these small deviations to variation in the optical properties over the film thickness that is not captured fully by the ellipsometry ${ }^{35}$, which provides only the average film properties.

\section{Optically induced phase transition of antenna- $\mathrm{VO}_{2}$ hybrids}

The reduction of the antenna OD above the phase transition is opposite to the effect of the $\mathrm{VO}_{2}$ film itself, where the OD is increased above the phase transition due to increased absorption in the switched metallic state, as shown in Supplementary Fig. S1. The spectral response shows an isosbestic point (that is, wavelength of constant OD between the two states) at $\sim 1000 \mathrm{~nm}$, with an increasing IMT-induced OD at longer wavelengths. In the following, we choose a probe wavelength of $1600 \mathrm{~nm}$, sufficiently far from the isosbestic point, as representative for the dynamics of the $\mathrm{VO}_{2}$ film without antennas. The temperature-dependent $\mathrm{OD}$ of the $\mathrm{VO}_{2}$ film without antennas is presented in Figure $1 \mathrm{c}$. The $\mathrm{VO}_{2}$ film shows a typical hysteresis loop when the temperature is cycled between 25 and $80^{\circ} \mathrm{C}$, as indicated by the arrows. This hysteresis shows that thermal memory is present in the system, that is, the sample response depends on its thermal history.

Next to controlling the sample temperature, the phase transition could be triggered through optical pumping. For this purpose, we
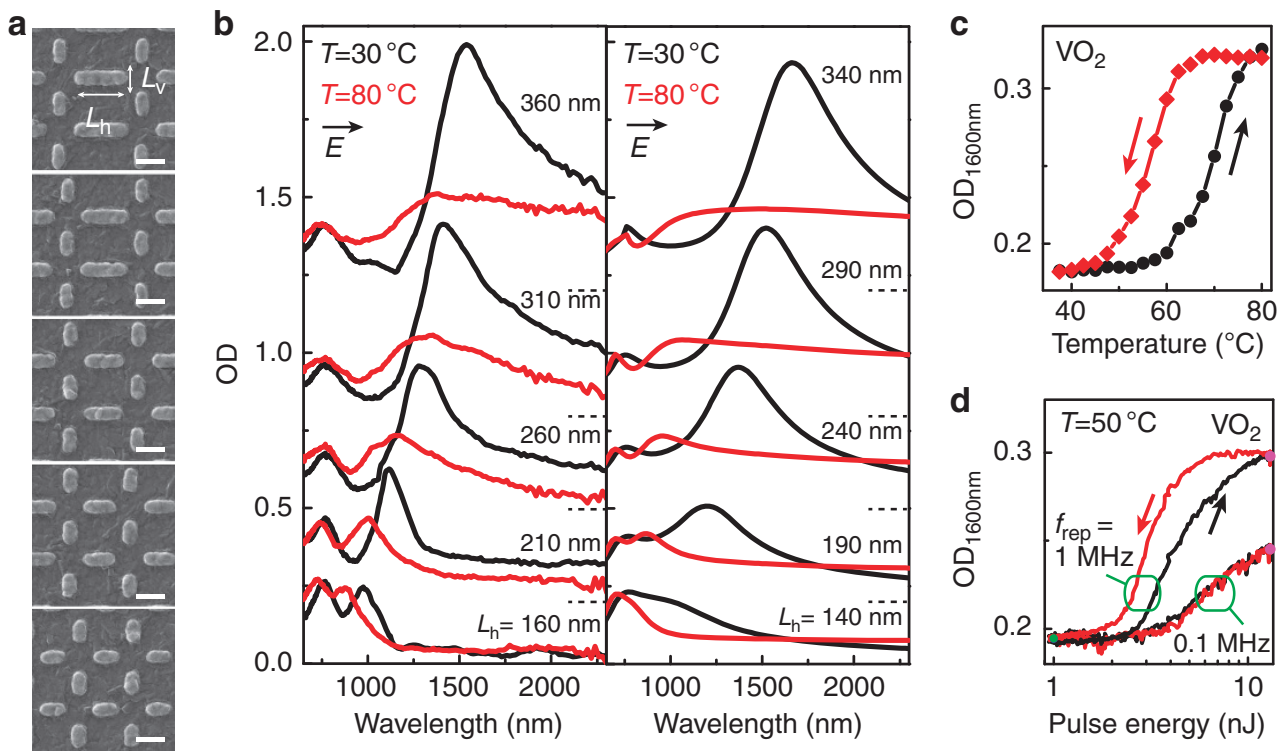

Figure 1 Temperature control of the resonances of the antenna- $\mathrm{VO}_{2}$ hybrids. (a) Helium-ion microscopy images of antenna arrays corresponding to fixed $L_{v}=160 \mathrm{~nm}$ and, from bottom to top, $L_{h}=160,210,260,310$ and $360 \mathrm{~nm}$. Scale bars, $200 \mathrm{~nm}$. (b) Experimental (left) and calculated (right) extinction spectra (OD) of antenna arrays corresponding to the images of a, for horizontal polarization. The response of the bare $\mathrm{VO}_{2}$ film and substrate without antennas was subtracted (see Supplementary Information). The sample temperature was set to $30{ }^{\circ} \mathrm{C}$ (black lines) and $80{ }^{\circ} \mathrm{C}$ (red lines). (c) Temperature hysteresis curve for the $\mathrm{OD}$ of bare $\mathrm{VO}_{2}$ film without antennas, taken at $\lambda_{\text {probe }}=1600 \mathrm{~nm}$. (d) Optical pumping hysteresis curve for bare $\mathrm{VO}_{2}$, for increasing (black) and decreasing (red) pump energies, taken at repetition rates of 1.0 and $0.1 \mathrm{MHz}$ and a pump wavelength $1060 \mathrm{~nm}$. 
fixed the sample temperature to $50{ }^{\circ} \mathrm{C}$, sufficiently below the hysteresis loop of the $\mathrm{VO}_{2}$ to allow full relaxation of the sample to its lowtemperature, unswitched state. The optical excitation makes use of a picosecond pulsed laser, as described in the Materials and Methods section, producing a train of pulses with variable repetition rate. To study the switching caused by individual pulses over a single cycle, as opposed to accumulated heating effects, it is important that the time between subsequent pump pulses is sufficiently long to allow the sample to recover its unperturbed state without a memory of previous events. Figure 1d shows the response of the $\mathrm{VO}_{2}$ film at $1600 \mathrm{~nm}$ under excitation by a train of picosecond pump pulses at a repetition rate of $1.0 \mathrm{MHz}$ and a pump wavelength of $1060 \mathrm{~nm}$. The OD was sampled at a pump-probe time delay of $100 \mathrm{ps}$ following pulsed laser excitation, corresponding to a fully developed phase transition. With increasing pulse energy, the OD increases to a level close to the thermal switching amplitude of Figure $1 \mathrm{c}$ at the highest available pump energy of $13 \mathrm{~nJ}$, indicating that a complete phase transition is achieved using optical pumping under these conditions. However, when ramping down the power, hysteresis behavior is observed. This hysteresis indicates that thermal memory is still present for pulsed excitation at a $1-\mathrm{MHz}$ repetition rate, and part of the response results from heat accumulated in the sample over subsequent excitation cycles. The hysteresis in the optical pumping cycle is completely suppressed when reducing the repetition rate to $0.1 \mathrm{MHz}$, where it is evident that single-cycle pulsed excitation only achieves approximately half of the modulation amplitude of Figure 1c.

The balance between fast, single-cycle switching and slow, accumulated heat effects is investigated in more detail in Figure 2 by studying the dependence on the optical pumping repetition rate. We used a pulse picker to reduce the laser repetition rate in steps from 10 to $0.1 \mathrm{MHz}$. The top panel of Figure 2a shows the differential transmission $\Delta \mathrm{OD} / \mathrm{OD}_{\mathrm{VO}_{2}}$ against the pump-probe delay time for the bare $\mathrm{VO}_{2}$ film without antennas. The bottom panel in Figure 2a shows the response measured for the antenna- $\mathrm{VO}_{2}$ hybrid array with $L_{\mathrm{v}}=L_{\mathrm{h}}=210 \mathrm{~nm}$ at the antenna resonance $\left(\lambda_{\text {probe }}=1200 \mathrm{~nm}\right)$, normalized to the maximum $\mathrm{OD}$ of the antenna, $\mathrm{OD}_{\mathrm{Ant}}=0.4$ (see Supplementary Fig. S4). The pump energy of $P_{\text {pump }}=0.2 \mathrm{~nJ}$ was limited by the damage threshold at the highest repetition rates. The relevant dynamics did not depend on the pulse energy.

A picosecond, fast component is identified by the step in the $\Delta \mathrm{OD} /$ OD response at $\sim 0 \mathrm{ps}$. At high-repetition rates, the amplitude of this fast, picosecond component is reduced, whereas instead the $\Delta \mathrm{OD} / \mathrm{OD}$ response is governed by a flat, stationary response that does not depend on the picosecond dynamics. The balance between these two effects is best illustrated by plotting the relative contributions to the total response shown in Figure $2 b$, where the contributions are defined as $\Delta \mathrm{OD}_{\text {slow }} / \Delta \mathrm{OD}$ and $\Delta \mathrm{OD}_{\text {fast }} / \Delta \mathrm{OD}$. Here $\Delta \mathrm{OD}$ is the total change, and $\Delta \mathrm{OD}_{\text {slow }}$ and $\Delta \mathrm{OD}_{\text {fast }}$ are the slow and fast components, identified by the $\Delta \mathrm{OD}$ before and after $0 \mathrm{ps}$, respectively. For lowrepetition rates, the response is entirely governed by the fast, picosecond component corresponding to excitation by a singlepump pulse at every cycle. At higher repetition rates above a critical threshold (indicated by the vertical line), the stationary thermal background dominates, and the fast, picosecond component is suppressed to zero. The stationary background corresponds to an increased temperature of the sample around the excitation area, which is built up over many pumping cycles because the sample is not completely relaxed between subsequent pulses. Because both the background and picosecond effects act on the same IMT, an increase of the slow effect results in suppression of the fast component because a
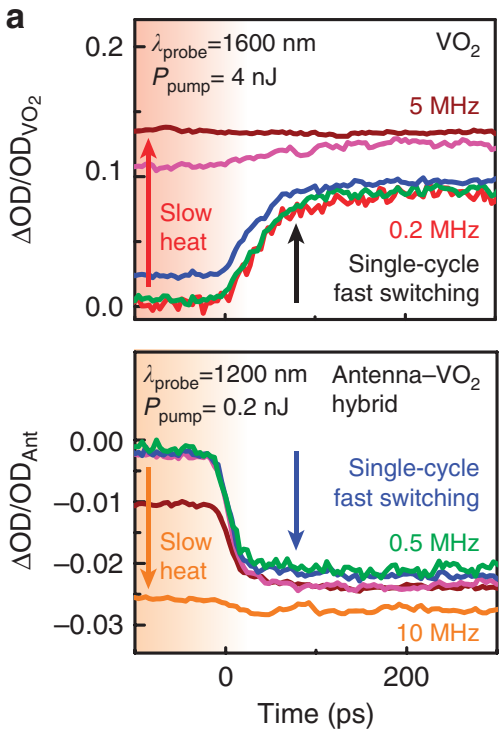

b
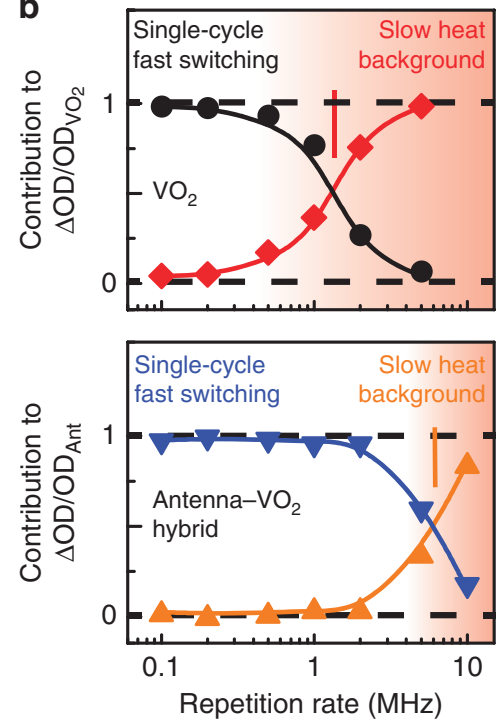

C

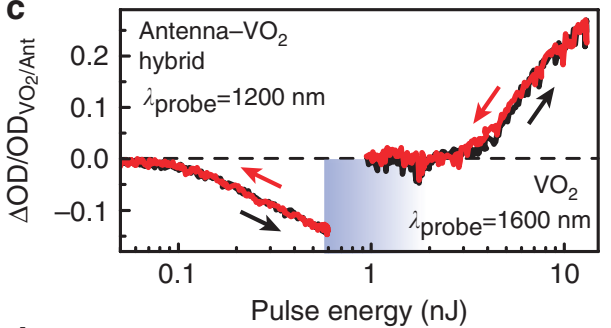

d

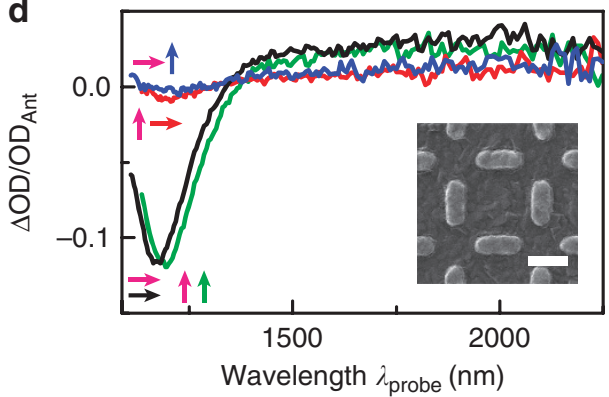

Figure 2 Contributions of the fast picosecond and slow thermal response to the optically driven $\mathrm{IMT}_{\mathrm{M}}$ in $\mathrm{VO}_{2}$ and antenna-VO $\mathrm{O}_{2}$ hybrids. (a) Time-dependent response of $\mathrm{VO}_{2}$ (top, $\lambda_{\text {probe }}=1600 \mathrm{~nm}$ ) and antenna array with $L_{\mathrm{v}}=L_{\mathrm{h}}=210 \mathrm{~nm}$ (bottom, $\lambda_{\text {probe }}=1200 \mathrm{~nm}$ ) for repetition rates of $0.2 \mathrm{MHz}($ red), $0.5 \mathrm{MHz}$ (green), $1 \mathrm{MHz}$ (blue), $2 \mathrm{MHz}$ (magenta), $5 \mathrm{MHz}$ (brown) and $10 \mathrm{MHz}$ (orange). The effect before 0 ps corresponds to a slow heat background, with the step at 0 ps corresponding to single-cycle fast switching caused by picosecond phase transition. Pump energy $P_{\text {pump }}$ for the VO $\mathrm{O}_{2}$ film, $4 \mathrm{~nJ}$; for the antenna array, $0.2 \mathrm{~nJ}$. (b) Relative contributions of single-cycle fast $\Delta \mathrm{OD}_{\text {fast }} / \Delta \mathrm{OD}$ and slow heat background $\Delta O \mathrm{D}_{\text {slow }} / \Delta \mathrm{OD}$ (see text) obtained from the time traces in a. The antenna array shows $5 \times$ increased operating speed for single-cycle fast switching compared with pure $\mathrm{VO}_{2}$. (c) $\mathrm{Phase-change} \mathrm{modulation} \Delta \mathrm{OD} / \mathrm{OD}$ versus optical pulse energy at $\lambda_{\text {probe }}=1200 \mathrm{~nm}$ for the antenna array and at $\lambda_{\text {probe }}=1600 \mathrm{~nm}$ for the $\mathrm{VO}_{2}$ film, both at $0.1 \mathrm{MHz}$. The blue shaded region indicates the damage threshold for the antenna array. (d) Differential nonlinear response spectrum $\Delta O D / O D_{\text {Ant }}$ for an antenna array with $L_{v}=L_{h}=210$ nm, for different combinations of pump (magenta arrows) and readout (black/red arrows, horizontal, green/blue arrows, vertical) polarizations, at a temperature of $50{ }^{\circ} \mathrm{C}$, $0.1 \mathrm{MHz}$ pump laser repetition rate, and $0.6 \mathrm{~nJ}$ pump energy. 
the sample is maintained in the switched state by the accumulated heat. For the $\mathrm{VO}_{2}$ film without antennas, this crossover from a fast picosecond response (black dots) to the slow heat background signal (red diamonds) occurs at an $\sim 1 \mathrm{MHz}$ repetition rate, indicating that the typical recovery time of the system is $\sim 1 \mu \mathrm{s}$. For the antenna- $\mathrm{VO}_{2}$ hybrid system, the crossover from picosecond to slow heat background response occurs at $\sim 5 \mathrm{MHz}$, five times higher frequency than for the $\mathrm{VO}_{2}$ film. Thus, the typical recovery time of the hybrid system is $\sim 200 \mathrm{~ns}$.

The response of the antenna- $\mathrm{VO}_{2}$ hybrid is characterized by a strong reduction in the optical switching energy. Figure $2 \mathrm{c}$ compares $\Delta \mathrm{OD} / \mathrm{OD}_{\text {Ant }}$ for the $L_{\mathrm{v}}=L_{\mathrm{h}}=210 \mathrm{~nm}$ antenna- $\mathrm{VO}_{2}$ hybrid with $\Delta \mathrm{OD} / \mathrm{OD}_{\mathrm{VO}_{2}}$ of the bare $\mathrm{VO}_{2}$ film against pulse energy at a repetition rate of $0.1 \mathrm{MHz}$. While the bare $\mathrm{VO}_{2}$ shows an onset of IMT switching for amplitudes $>2 \mathrm{~nJ}$, the antenna- $\mathrm{VO}_{2}$ hybrids show an onset at $\sim 100 \mathrm{pJ}$, which is a 20 times lower pulse energy. Irreversible damage to the antenna- $\mathrm{VO}_{2}$ hybrids is caused above $800 \mathrm{pJ}$, limiting the maximum modulation for this configuration to $\sim 15 \%$. The $\mathrm{VO}_{2}$ film itself reaches similar modulation contrast values of up to $20 \%$; however, this requires a much higher pulse energy of $13 \mathrm{~nJ}$.

\section{Antenna-assisted resonant pumping of the phase transition}

The fivefold reduction in thermal recovery time and $>20$ times lower switching energy of the antenna- $\mathrm{VO}_{2}$ hybrids suggests a new excitation pathway distinct from bulk switching of the $\mathrm{VO}_{2}$ film without antennas. To further explore the contribution of antenna-assisted mechanisms in the antenna- $\mathrm{VO}_{2}$ hybrid response, we measured the switching response while independently controlling the polarizations of both excitation and readout. This strategy was used successfully in previous studies for antennas on an ITO substrate ${ }^{36}$. Figure $2 \mathrm{~d}$ shows the spectrally resolved $\triangle \mathrm{OD}$ normalized to $\mathrm{OD}_{\text {Ant }}$ measured on the $L_{\mathrm{v}, \mathrm{h}}=210 \mathrm{~nm}$ antenna- $\mathrm{VO}_{2}$ hybrid array for the four different combinations of pump and probe polarizations, as indicated by the pairs of arrows, with the first (magenta) and second (colored) arrow, indicating pump and probe polarizations, respectively. The pumping was performed at a $0.1 \mathrm{MHz}$ repetition rate and $0.6 \mathrm{~nJ}$ energy at $1060 \mathrm{~nm}$ pump wavelength. A large modulation amplitude is observed only for the condition, where the pump and readout polarizations are parallel to each other. For perpendicular polarizations, no switching response is observed. Given that perpendicularly oriented antennas are positioned within $100 \mathrm{~nm}$ from each other in the array, the lack of cross-interaction of the antenna-mediated IMT is remarkable and shows experimentally that the induced effects are limited to only a nanoscale-sized volume around the antenna- $\mathrm{VO}_{2}$ hybrids, which can be specifically addressed by the combination of the polarization and wavelength of the excitation laser.

Experiments performed on different arrays of antenna- $\mathrm{VO}_{2}$ hybrids, corresponding to different combinations of lengths $L_{\mathrm{h}}$ and $L_{v}$, show the response when tuning the system from resonant to nonresonant pumping conditions. Figure 3a shows the results for arrays of crossed antennas with equal arm lengths $L_{\mathrm{h}}$ and $L_{\mathrm{v}}$ tuned (bottom to top) from 160 to $360 \mathrm{~nm}$. The polarization-dependent response is shown for the pump and readout polarizations that are parallel (black curves) and perpendicular (red curves). The strong IMT response around the plasmon resonance for parallel polarizations, which is most clearly observed for the 210-nm length antennas corresponding to Figure 2d, disappears with increasing arm length. For crossed a

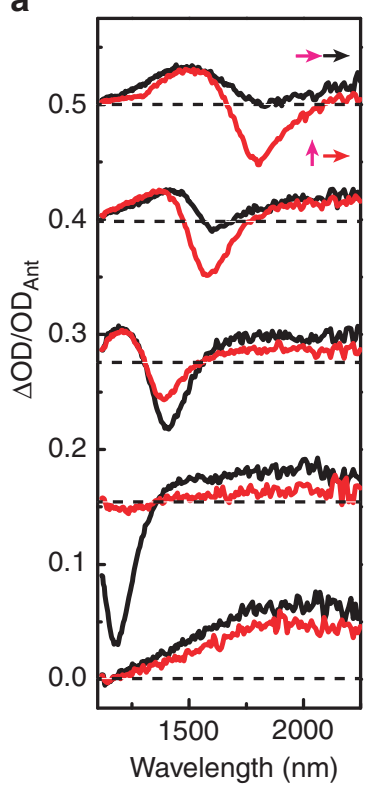

b

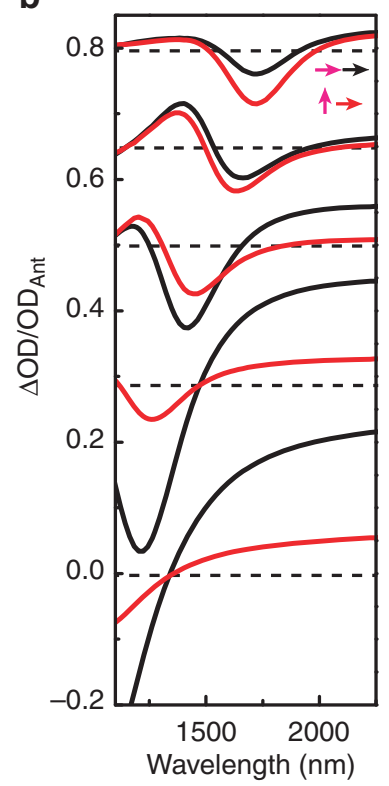

C

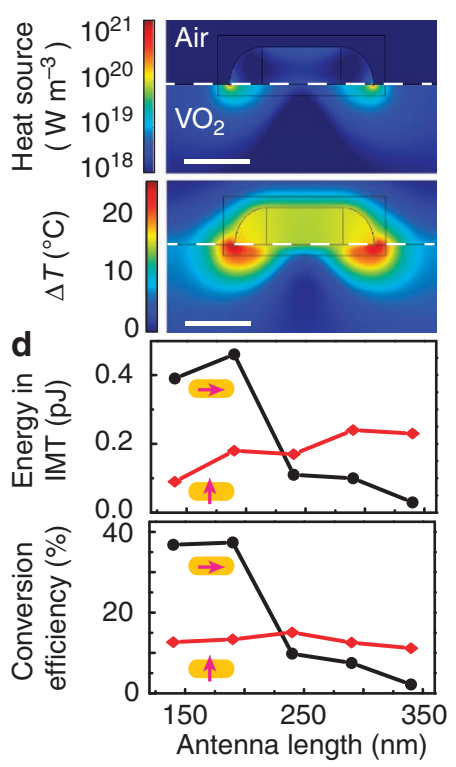

e

Temperature iso-surface in $\mathrm{VO}_{2}$ $T=68^{\circ} \mathrm{C}$

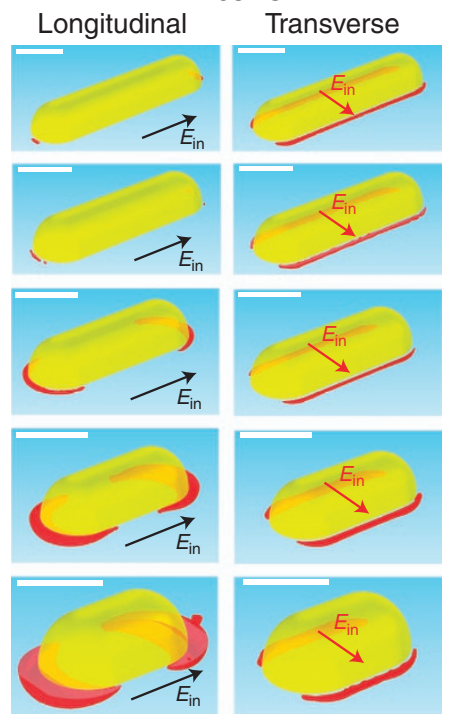

Figure 3 The model shows that the predominant phase-change response is caused by antenna-assisted absorption in the $\mathrm{VO}_{2}$ at plasmonic hotspots. Experimental (a) and calculated (b) switching response $\triangle \mathrm{OD} / \mathrm{OD}_{\text {Ant }}$ of the antenna- $\mathrm{VO}_{2}$ hybrids for parallel (black lines) and perpendicular (red lines) polarizations for excitation and readout for (bottom to top) antenna arm lengths $L_{h}=L_{v}=160,210,260,310$ and $360 \mathrm{~nm}$ (calculated antennas are $20 \mathrm{~nm}$ shorter). Dashed lines indicate vertical offsets. (c) Calculated heat source distribution (top panel) in antenna- $\mathrm{VO}_{2}$ hybrid with $190 \mathrm{~nm}$ antenna length during the pump laser pulse, calculated using the full electrodynamic model of local absorption, and the temperature increase $\Delta T$ calculated by including the timedependent heat diffusion model for 9.2-ps pump duration. Scale bars, $100 \mathrm{~nm}$. (d) Calculated energy contained in the IMT regions and conversion efficiency (\% of incident energy) versus antenna length and pump polarization (arrows) for a single antenna. (e) Temperature iso-surfaces for the critical phase transition temperature, $T=68{ }^{\circ} \mathrm{C}$, for antenna lengths from 160 to $360 \mathrm{~nm}$ (bottom to top) and longitudinal (left row) and transverse (right row) polarizations. Scale bars, $100 \mathrm{~nm}$. 
polarizations, the nonlinear $\Delta \mathrm{OD}$ response increases as the antenna length is increased.

\section{Model of antenna-assisted phase transition}

These trends are reproduced well by the detailed numerical calculations plotted in Figure $3 \mathrm{~b}$. The physical model underlying these calculations is presented in Figure 3c-3e. First, the near-field absorption profile around the antenna was calculated based on full electrodynamic calculations of the near-field intensity. The top panel of Figure 3c shows resulting maps for an $L=190 \mathrm{~nm}$ antenna and incident light polarized along the antenna axis, for an excitation power corresponding to an integrated energy of $300 \mathrm{pJ}$ (fluence of $2.75 \mathrm{~mJ} \mathrm{~cm}^{-2}$ ) over the pulse duration. The calculated local absorption profile was used as a quasi-stationary heat source distribution for the heat-transport equation, resulting in the temperature profile shown in the bottom panel of Figure 3c. To separate the different contributions to the total temperature rise originating from energy absorbed in the gold antenna and in the $\mathrm{VO}_{2}$ film, we independently evaluated the role of the antenna as a heat source (results shown in the Supplementary Fig. S8), and found that absorption outside the plasmonic antenna dominates the heat source distribution under conditions of resonant pumping and nearly completely explains the temperature increase without requiring additional energy transport from inside the nanoparticle into the $\mathrm{VO}_{2}$ layer.

From the calculated local temperature profile, nanoscale volumes can be extracted, in which the temperature is sufficiently high to induce the $\mathrm{VO}_{2} \mathrm{IMT}$. Figure $3 \mathrm{e}$ shows the resulting contours, plotted in red, for antennas with lengths increasing from 140 to $340 \mathrm{~nm}$ (the length of the model antennas corresponds to the experimental cases from 160 to $360 \mathrm{~nm}$ ). Both the longitudinal and transverse pump polarizations are shown for each antenna. For longitudinal pump polarization, resonant pumping results in pronounced pockets of IMT response around the end caps of the antenna for the shortest antennas. For longer antenna lengths, the effect of resonance is weaker, and the critical temperature threshold is reached within a much smaller volume. In comparison, the transverse pump polarization shows approximately the same effect for all antenna lengths. This contribution is caused by off-resonance absorption of the transverse plasmon mode, which causes a narrow band of IMT that increases linearly in length as the antenna length is increased.

The optical spectra in Figure $3 \mathrm{~b}$ were obtained by including the calculated IMT volumes in the electrodynamic simulation, using the known dielectric response of the switched $\mathrm{VO}_{2}$. Our microscopic model satisfactorily explains the two trends for the longitudinal and transverse excitation conditions, corresponding to the resonant pumping of the IMT for longitudinal polarization and the nonresonant increase for transverse polarization. The model predicts an effective switching energy of up to $0.46 \mathrm{pJ}$ per antenna at resonance, as shown in Figure 3d. Up to $39 \%$ of the total absorbed energy is converted into the $\mathrm{VO}_{2}$ phase transition, making the resonant, antenna-assisted absorption mechanism a potentially highly efficient process for device applications. For the pumping of the transverse resonance, the model calculates energy conversion efficiencies in the range of $12-15 \%$.

The results in Figure 3 show that the switching spectra for crossantenna arrays with equal lengths can be explained using the antennaassisted IMT response of single antennas without cross-coupling. The case of unequal antenna lengths is investigated in Figure 4. Figure $4 \mathrm{a}$
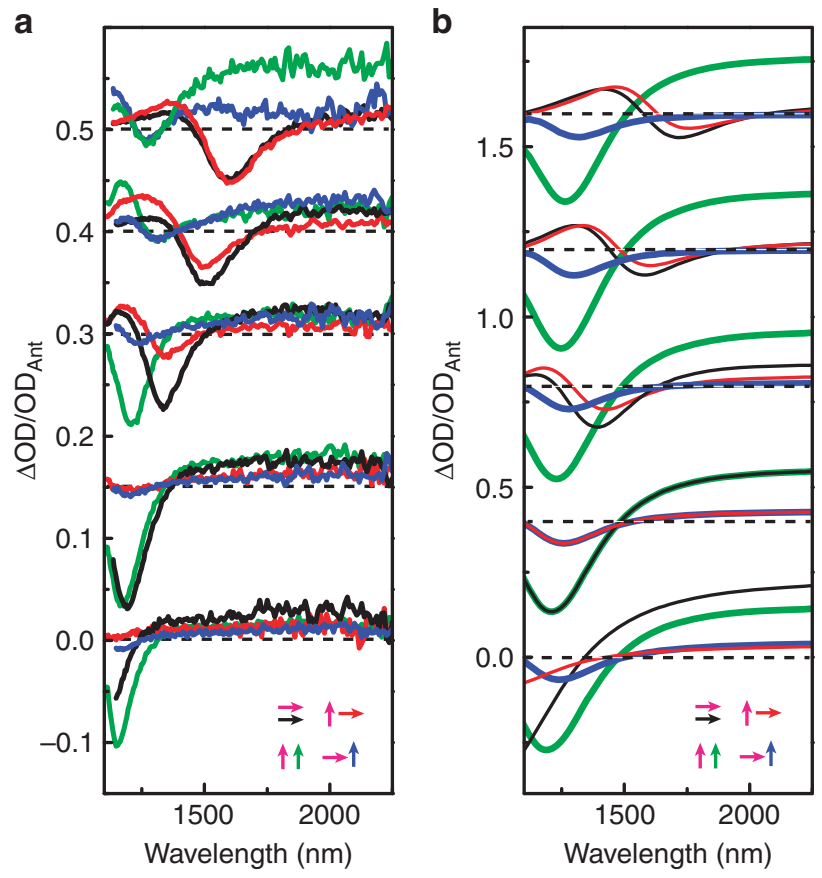

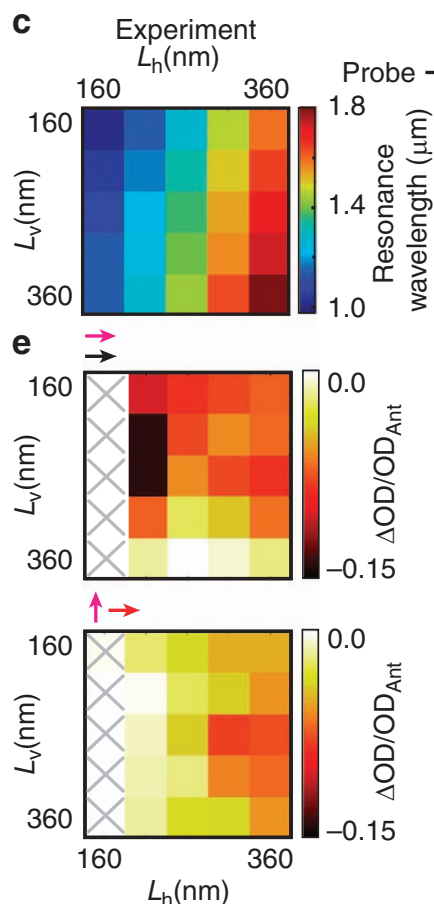

d Calculation
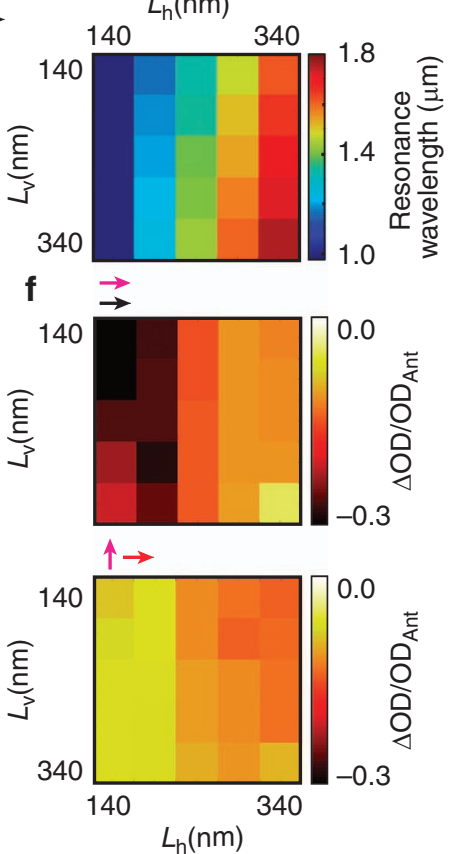

Figure 4 Full investigation of the multifrequency antenna- $\mathrm{VO}_{2}$ hybrids shows resonant contribution in antenna-mediated IMT response. (a) Experimental and (b) calculated switching response $\Delta O D / O D_{\text {Ant }}$ of antenna- $\mathrm{VO}_{2}$ hybrids for fixed $L_{v}=210 \mathrm{~nm}$ and, bottom to top, varying $L_{\mathrm{h}}=160,210,260,310$ and $360 \mathrm{~nm}$ (calculated antennas are $20 \mathrm{~nm}$ shorter). Arrows indicate pump (magenta) and probe (black/red arrows, horizontal, green/blue arrows, vertical) polarizations. (c and d) Experimental (c) and calculated (d) resonance positions of the horizontal (top panel) antennas in multifrequency antenna arrays with combinations of $L_{\mathrm{h}}$ and $L_{\mathrm{v}}$ (see Supplementary Fig. S2 for helium-ion microscopy images). (e and f) Experimental (e) and calculated (f) normalized amplitude $\Delta \mathrm{OD} / \mathrm{OD}_{\text {Ant }}$ for the horizontal readout (probe, black/red arrows) and for two pump polarizations, as indicated by magenta arrows. Crosses indicate arrays for which the resonance lies outside the spectral window of the probe. 
shows the full polarization response for four combinations of pump and probe polarizations for antennas with fixed $L_{\mathrm{v}}=210 \mathrm{~nm}$ and $L_{\mathrm{h}}$ varying from 160 to $360 \mathrm{~nm}$. Each $\Delta \mathrm{OD}$ spectrum was normalized to the $\mathrm{OD}_{\mathrm{Ant}}$ of the corresponding antenna resonance. The corresponding calculated response is shown in Figure $4 \mathrm{~b}$. Both experiment and theory show good agreement in the spectral positions and line shapes of the $\Delta \mathrm{OD}$ switching response, resembling that of the uncoupled single rods. The only deviation is for the resonant excitation of the fixed vertical antenna (green curves), which shows a suppression and change in shape for the longest horizontal antenna lengths.

The global trends over the $25(5 \times 5)$ different arrays are illustrated by the color maps shown in Figure $4 \mathrm{c}-4 \mathrm{f}$. Figure $4 \mathrm{c}$ and $4 \mathrm{~d}$ shows the spectral resonance positions extracted from the linear extinction spectra (see Supplementary Figs. S4 and S6). The maximum negative $\Delta \mathrm{OD} / \mathrm{OD}_{\text {Ant }}$ amplitudes are shown in Figure $4 \mathrm{e}$ and $4 \mathrm{f}$ for two of the polarization combinations. The other two polarization cases can be obtained by mirroring the matrices along the diagonal. For the shortest 160-nm length antennas, the resonance lies below the accessible spectral range; therefore, we discarded these data points (crossed out).

The diagonal components of the matrices correspond to the equal length antenna arrays in Figure $3 \mathrm{a}$ and $3 \mathrm{~b}$, and show good agreement between experiment and theory. For the off-diagonal components, the transverse (cross-polarized) pumping conditions are reproduced for all combinations and show little dependence on the ratio of the horizontal and vertical antenna lengths (vertical bands of equal modulation intensity). For the longitudinal excitation (parallel polarizations), much stronger co-dependence on the antenna parameters is observed for the experimental case than for the calculations, as was observed in Figure 4a. The strongest overall modulation response is found for the $L_{\mathrm{v}}=L_{\mathrm{h}}=210 \mathrm{~nm}$ array, and the presence of longer antennas in the cross-direction reduces the modulation depth in the experimental data but does not affect the calculated response. Thus, the experimental data indicate stronger cross-interaction effects between antennas, which are not yet fully captured by our model. These variations correspond to subtle differences in the microscopic energy distribution, which may be fine-tuned in future work. Overall, we find that the simple localized absorption picture provides a good qualitative and semi-quantitative description of the observed behavior.

\section{Thermal latching optical memory of antenna- $\mathrm{VO}_{2}$ hybrids}

The above investigation shows that it is possible to achieve local IMT switching through resonant optical pumping of $\mathrm{VO}_{2}$ using nanoscale hotspots. The local pumping significantly reduces the required optical power, thermal load and recovery time of the device. These characteristics are of interest for applications in optical memory, memristors, switches and modulators. The memory functionality of antenna- $\mathrm{VO}_{2}$ hybrids is based on the thermal hysteresis characteristics, which we minimized by using pulsed laser excitation at sub-MHz repetition rates. However, memory functionality can be introduced by raising the base temperature into the hysteresis regime ${ }^{28}$. Figure $5 \mathrm{a}$ shows the dependence of the optically induced state on the base sample temperature, as set by the external heater stage. At room temperature, picosecond optical switching is fully reversible and can be cycled over millions of times per second. At elevated temperatures above $55^{\circ} \mathrm{C}$, the system is latched to a value
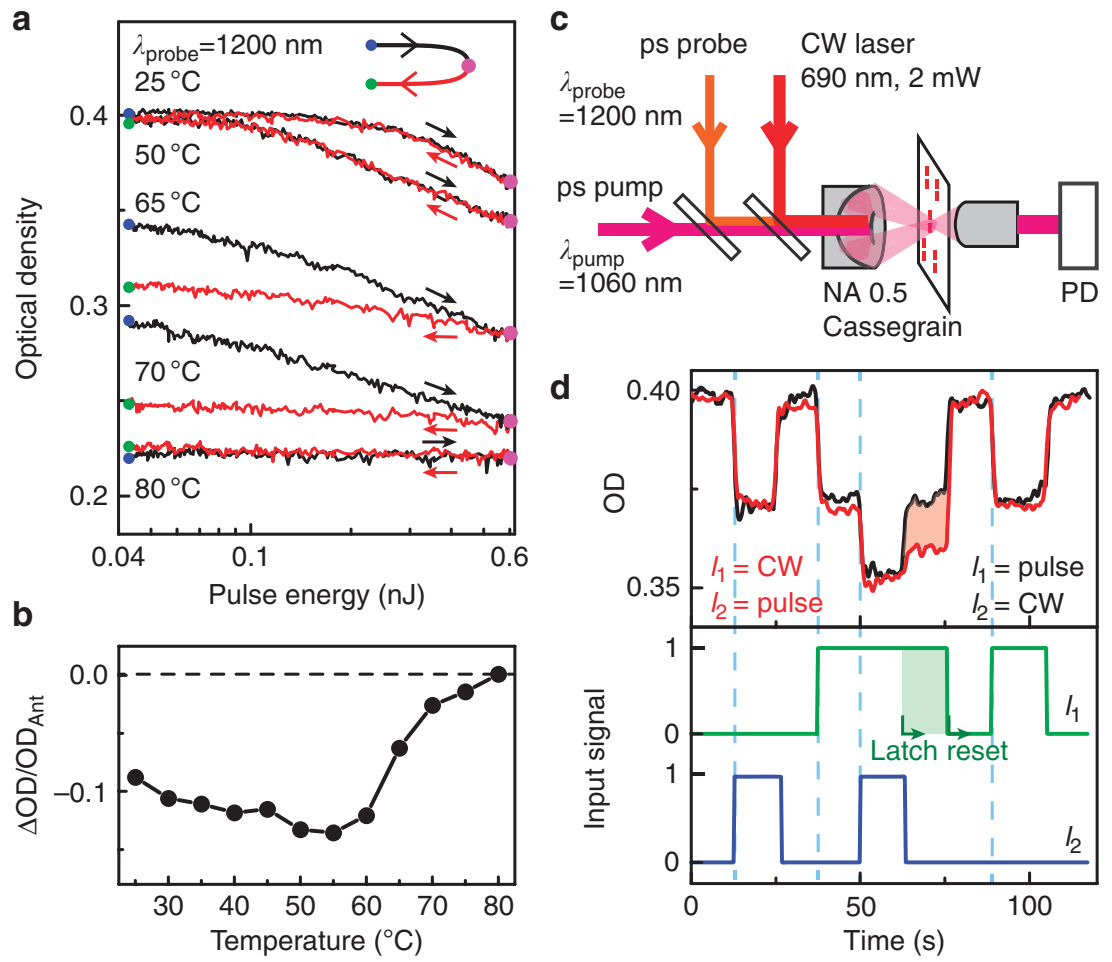

Figure 5 Thermal latching and memory functionality of antenna- $\mathrm{VO}_{2}$ hybrids. (a) Optical density of the antenna-VO $\mathrm{O}_{2}$ hybrid with $L_{\mathrm{V}}=L_{\mathrm{h}}=210 \mathrm{~nm}$ while scanning the pulse energy up (black line) and down (red line) for background temperatures from 50 to $80{ }^{\circ} \mathrm{C}$. Data were taken at $1200 \mathrm{~nm}$ and for parallel pump and readout polarizations at $0.1 \mathrm{MHz}$. (b) Differential response $\Delta O D / O D_{\text {Ant }}$ versus background temperature for the same experimental conditions as in a, for $P_{\text {pump }}=0.6 \mathrm{~nJ}$. (c) Experimental setup for the combined pulsed and CW pumping experiment. (d) Combined effect of two input pump sequences corresponding to 1 ps and one CW input. Sequence shows individual effects of both input $I_{1}$ and $I_{2}$ (bottom panel) and the combined effect of the two signals, including a memory latch (indicated by shaded area). Memory functionality is only observed for the CW control input $I_{1}$, whereas picosecond pulses do not provide latching functionality. 
determined by the downward branch of the hysteresis loop of Figure 1c. This latching effect for the case of resonant plasmonic pumping is nontrivial because it involves maintaining the integrity of the small, nanoscale pockets of switched $\mathrm{VO}_{2}$ around the antenna end caps. In our experiments, we did not observe degradation of the latched state over typical time scales of minutes. At sample temperatures above the IMT $\left(80^{\circ} \mathrm{C}\right.$ in Figure $\left.5 \mathrm{a}\right)$, optical pumping does not induce further effects because the whole sample is already switched thermally by the heater stage.

In the differential $\Delta \mathrm{OD} / \mathrm{OD}_{\mathrm{Ant}}$ response (Figure $5 \mathrm{~b}$ ), taken at the pulse energy of $0.6 \mathrm{~nJ}$, the thermal latching effect is observed as a reduction of the picosecond transient signal to zero as the system crosses the hysteresis regime of the IMT. Furthermore, while the largest signal amplitudes are obtained just below the hysteresis regime at $\sim 50{ }^{\circ} \mathrm{C}$, a modulation response is obtained all the way down to room temperature, showing that operation of antenna- $\mathrm{VO}_{2}$ hybrids for picosecond switching is possible without requiring an elevated base temperature.

In addition to setting the base temperature, latching of the phase transition could also be achieved through continuous-wave optical pumping of the sample. Figure $5 \mathrm{c}$ shows the experimental arrangement, where a continuous wave $(\mathrm{CW})$ diode laser at a wavelength of $690 \mathrm{~nm}$ was used to achieve continuous thermal excitation at a $\Delta \mathrm{OD}$ level comparable to that of the picosecond laser-induced response. We tested the latching effect using two pulse sequences, where the $\mathrm{CW}$ and picosecond pulsed pump lasers were combined to provide an additive effect on the IMT response of the readout beam. In the first scheme, intensity sequence $I_{1}$ was applied to the CW laser and sequence $I_{2}$ was applied to the picosecond laser. In this sequence, the switch was prepared in the trigger state at $40 \mathrm{~s}$ using the CW laser $\left(I_{1}\right)$, after which the memory was triggered by the picosecond laser $\left(I_{2}\right)$ at $50 \mathrm{~s}$. When the picosecond signal $I_{2}$ was switched off at $65 \mathrm{~s}$, the system remained in the latched state until the removal of the CW control signal at $80 \mathrm{~s}$. Similar to thermal latching using the heater stage, the optically latched state was very stable over minutes, and the fluctuations in Figure $5 \mathrm{~d}$ mainly reflect stability of the microscope setup itself. When $I_{1}$ and $I_{2}$ are reversed, that is, $I_{1}$ corresponds to the single-shot reversible excitation, this latching effect is no longer obtained because the base temperature is not retained for pulsed excitation. Thus, picosecond pulsed excitation and CW thermal excitation with the same optical response provide different memory functionalities and can be used in combination to achieve different switching operations.

\section{Discussion}

It is of interest to compare our results obtained in the picosecond regime with other works using continuous-wave or ultrafast (sub-100 fs) pulsed excitation. The critical pulse energies for the antenna- $\mathrm{VO}_{2}$ hybrid and the bulk $\mathrm{VO}_{2}$ translate to fluences of $\sim 0.9 \mathrm{~mJ} \mathrm{~cm}^{-2}$ and $18 \mathrm{~mJ} \mathrm{~cm}^{-2}$, respectively. Continuous-wave experiments show a threshold for the $\mathrm{VO}_{2}$ IMT of $\sim 100 \mathrm{~mW} \mathrm{~cm}^{-2}$ (Refs. 28 and 33). However, this mechanism involves slow heating of both the $\mathrm{VO}_{2}$ and substrate to the critical temperature over hundreds of milliseconds ${ }^{33}$. For sub-100 fs excitation, the critical fluence amounts to $4.7 \mathrm{~mJ} \mathrm{~cm}{ }^{-2}$ for bulk $\mathrm{VO}_{2}{ }^{18,39,40}$. The fluences in our work are therefore higher than the values reported for nonthermal excitation of $\mathrm{VO}_{2}$ domains, which benefit from highly nonequilibrium effects to cross the energy barrier for the IMT. While the use of picosecond pulses does not benefit from the nonequilibrium physics of ultrafast lasers, there is a strong motivation for device applications to exploit efficient picosecond mechanisms for optical devices, such as those based on the fast thermal response of plasmonic nanoantennas ${ }^{34}$. Because the pump area contains $\sim 60$ of the resonant antennas, the experimental switching energies between 100 and $600 \mathrm{pJ}$ correspond to 1.6 and $10.0 \mathrm{pJ}$ per antenna, closely matching our numerical model calculations. Thus, picojoule single-antenna switches are feasible using this approach.

In comparison, the reported switching fluence of chalcogenide phase-change materials using femtosecond laser pulses is $140 \mathrm{~mJ} \mathrm{~cm}{ }^{-2}$ (Ref. 13), corresponding to $1.5 \mathrm{~nJ}$ pulse energy for a full set-reset cycle. In particular, the reset pulse in non-volatile GST phase-change memory locally exposes the device to temperatures above the melting point of $620^{\circ} \mathrm{C}$, resulting in a significant thermal load and crosstalk, which can pose limitations in practical applications. In contrast, the temperature in picosecond antenna- $\mathrm{VO}_{2}$ hybrids never rises far above the phase transition of $68^{\circ} \mathrm{C}$ and is therefore friendlier toward integration into nanophotonic devices. Given that a single picojoule pulse induces persistent IMT switching for several hundreds of nanoseconds, the average power requirement to sustain a singleantenna- $-\mathrm{VO}_{2}$ hybrid memory can be estimated as $\sim 10-100 \mu \mathrm{W}$. Thus, antenna- $\mathrm{VO}_{2}$ hybrids are expected to be competitive with GST phase change, especially for short-term memory applications with cycling times shorter than $1 \mathrm{~ms}$.

In addition to the thermally mediated phase transition, recent studies have proposed contributions of ultrafast plasmon-induced hot electrons $s^{31,32}$. These additional effects are more likely to play a role for much shorter laser pulses in the $100 \mathrm{fs}$ range, that is, much shorter than the 9.2 ps pump laser used in this work. Our modeling based on the measured optical constants predicts absorption primarily in the $\mathrm{VO}_{2}$ itself and reproduces our experimental results without requiring the additional mechanism of hot-electron excitation and escape through the gold- $\mathrm{VO}_{2}$ interface. Therefore, we have not considered these effects in our interpretation.

\section{CONCLUSION}

We have demonstrated that resonant pumping of plasmonic nanoantenna- $\mathrm{VO}_{2}$ hybrids results in high-repetition, fully reversible switching of the insulator-to-metal phase transition. The use of plasmonic nearfield enhancement and hybrid antenna- $\mathrm{VO}_{2}$ response results in an order of magnitude lower switching energy and faster cycling time than bulk $\mathrm{VO}_{2}$ without antennas. Moreover, the antenna-assisted process allows precise, local control of the $\mathrm{VO}_{2}$ optical properties down to the nanoscale. These properties render antenna- $\mathrm{VO}_{2}$ hybrids of great interest for application in optical modulation and fast picosecond control of nanoscale devices. As a result of the large difference in switching energy between antenna-mediated and bulk $\mathrm{VO}_{2}$, the antenna- $\mathrm{VO}_{2}$ hybrid response can be selectively excited without inducing a background response from the $\mathrm{VO}_{2}$ film itself. Optical experiments show that fully reversible switching of antenna resonances with over two million cycles per second is possible using resonant pumping schemes. The insulator-to-metal phase transition mediated by local pumping of a plasmon resonance does not influence the resonance of a perpendicular nanoantenna positioned $<100 \mathrm{~nm}$ from the modulated antenna. Further improvement of the response may include optimization of the design, including selective deposition of $\mathrm{VO}_{2}$ in the direct surroundings of the antenna, embedding of antennas inside a $\mathrm{VO}_{2}$ matrix and improved cooling using substrates and coatings with high-thermal conductivity.

\section{CONFLICT OF INTEREST}

The authors declare no conflict of interest. 


\section{ACKNOWLEDGEMENTS}

We acknowledge $\mathrm{M}$ Abb for valuable contributions to the experimental setup and $\mathrm{H}$ Yates for stimulating discussions and support in the development of the $\mathrm{VO}_{2}$ deposition. This work was financially supported by EPSRC through research grant EP/J011797/1. OLM acknowledges support through an EPSRC Early Career Fellowship EP/J016918/1. LB, NZ and JA acknowledge financial support from Project No. FIS2013-41184-P of the Spanish Ministry of Economy and Competitiveness, project ETORTEK IE14-393 NANOGUNE'14 of the Department of Industry of the Government of the Basque Country, and support from the Basque Department of Education and the UPV-EHU (Grant No. IT-756-13). All data supporting this study are openly available from the University of Southampton repository at http://dx.doi.org/10.5258/SOTON/ 392922.

1 Zheludev NI, Kivshar YS. From metamaterials to metadevices. Nat Mater 2012; 11 : 917-924.

2 Schuller JA, Barnard ES, Cai W, Jun YC, White JS et al. Plasmonics for extreme light concentration and manipulation. Nat Mater 2010; 9: 193-204.

3 Strohfeldt N, Tittl A, Schäferling M, Neubrech F, Kreibig U et al. Yttrium hydride nanoantennas for active plasmonics. Nano Lett 2014; 14: 1140-1147.

4 Tittl A, Michel AKU, Schäferling M, Yin XH, Gholipour B et al. A switchable mid-infrared plasmonic perfect absorber with multispectral thermal imaging capability. Adv Mater 2015; 27: 4597-4603.

5 Sterl F, Strohfeldt N, Walter R, Griessen R, Tittl A et al. Magnesium as novel material for active plasmonics in the visible wavelength range. Nano Lett 2015; 15: 7949-7955.

6 Waters RF, Hobson PA, MacDonald KF, Zheludev NI. Optically switchable photonic metasurfaces. Appl Phys Lett 2015; 107: 081102.

7 Shportko K, Kremers S, Woda M, Lencer D, Robertson J et al. Resonant bonding in crystalline phase-change materials. Nat Mater 2008; 7: 653-658.

8 Gholipour B, Zhang JF, MacDonald KF, Hewak DW, Zheludev NI. An all-optical, nonvolatile, bidirectional, phase-change meta-switch. Adv Mater 2013; 25: 3050-3054.

9 Michel AKU, Chigrin DN, Maß TWW, Schönauer K, Salinga M et al. Using low-loss phase-change materials for mid-infrared antenna resonance switching. Nano Lett 2013; 13: 3470-3475.

10 Michel AKU, Zalden P, Chigrin DN, Wuttig M, Lindenberg AM et al. Reversible optical switching of infrared antenna resonances with ultrathin phase-change layers using femtosecond laser pulses. ACS Photonics 2014; 1: 833-839.

11 Yang Z, Ko C, Ramanathan S. Oxide electronics utilizing ultrafast metal-insulator transitions. Annu Rev Mater Res 2011; 41: 337-367.

12 Ríos C, Stegmaier M, Hosseini P, Wang D, Scherer T et al. Integrated all-photonic nonvolatile multi-level memory. Nat Photonics 2015; 9: 725-732.

13 Wang Q, Rogers ETF, Gholipour B, Wang C-M, Yuan GH et al. Optically reconfigurable metasurfaces and photonic devices based on phase change materials. Nat Photonics 2016; 10: 60-65.

14 Hosaka S, Shintani T, Miyamoto M, Kikukawa A, Hirotsune A et al. Phase change recording using a scanning near-field optical microscope. J Appl Phys 1996; 79: 8082-8086.

15 Kühler P, de Abajo FJG, Leiprecht P, Kolloch A, Solis J et al. Quantitative imaging of the optical near field. Opt Express 2012; 20: 22063-22078.

16 Qazilbash MM, Brehm M, Chae B-G, Ho P-C, Andreev GO et al. Mott transition in VO revealed by infrared spectroscopy and nano-imaging. Science 2007; 318: 1750-1753.

17 Cavalleri A, Tóth C, Siders CW, Squier JA, Ráksi F et al. Femtosecond structural dynamics in $\mathrm{VO}_{2}$ during an ultrafast solid-solid phase transition. Phys Rev Lett 2001; 87: 237401.

18 Wegkamp D, Stähler J. Ultrafast dynamics during the photoinduced phase transition in $\mathrm{VO}_{2}$. Prog Surf Sci 2015; 90: 464-502.

19 O'Callahan BT, Jones AC, Park JH, Cobden DH, Atkin JM et al. Inhomogeneity of the ultrafast insulator-to-metal transition dynamics of $\mathrm{VO}_{2}$. Nat Commun 2015; 6: 6849.
20 Driscoll T, Kim HT, Chae BG, Kim BJ, Lee YW et al. Memory metamaterials. Science 2009; 325: 1518-1521.

21 Liu MK, Hwang HY, Tao H, Strikwerda AC, Fan KB et al. Terahertz-field-induced insulator-to-metal transition in vanadium dioxide metamaterial. Nature 2012; 487: 345-348.

22 Seo M, Kyoung J, Park H, Koo S, Kim H-S et al. Active terahertz nanoantennas based on $\mathrm{VO}_{2}$ phase transition. Nano Lett 2010; 10: 2064-2068.

23 Thompson ZJ, Stickel A, Jeong J-G, Han S, Son BH et al. Terahertz-triggered phase transition and hysteresis narrowing in a nanoantenna patterned vanadium dioxide film. Nano Lett 2015; 15: 5893-5898.

24 Shelton DJ, Coffey KR, Boreman GD. Experimental demonstration of tunable phase in a thermochromic infrared-reflectarray metamaterial. Opt Express 2010; 18: 1330-1335.

25 Kats MA, Blanchard R, Genevet P, Yang Z, Qazilbash MM et al. Thermal tuning of mid-infrared plasmonic antenna arrays using a phase change material. Opt Lett 2013; 38: 368-370.

26 Dicken MJ, Aydin K, Pryce IM, Sweatlock LA, Boyd EM et al. Frequency tunable near-infrared metamaterials based on $\mathrm{VO}_{2}$ phase transition. Opt Express 2009; 17 18330-18339.

27 Son TV, Ba COF, Vallée R, Haché A. Nanometer-thick flat lens with adjustable focus. Appl Phys Lett 2014; 105: 231120.

28 Lei DY, Appavoo K, Ligmajer F, Sonnefraud Y, Haglund RF et al. Optically-triggered nanoscale memory effect in a hybrid plasmonic-phase changing nanostructure. ACS Photonics 2015; 2: 1306-1313.

29 Lei DY, Appavoo K, Sonnefraud Y, Haglund RF, Maier SA. Single-particle plasmon resonance spectroscopy of phase transition in vanadium dioxide. Opt Lett 2010; 35: 3988-3990.

30 Appavoo K, Haglund RF. Polarization selective phase-change nanomodulator. Sci Rep 2014; 4: 6771 .

31 Appavoo K, Wang B, Brady NF, Seo M, Nag J et al. Ultrafast phase transition via catastrophic phonon collapse driven by plasmonic hot-electron injection. Nano Lett 2014; 14: 1127-1133.

32 Hada M, Zhang DF, Casandruc A, Dwayne Miller RJ, Hontani Y et al. Hot electron injection driven phase transitions. Phys Rev B 2012; 86: 134101.

33 Ferrara DW, MacQuarrie ER, Nag J, Kaye AB, Haglund RF. Plasmon-enhanced low-intensity laser switching of gold: vanadium dioxide nanocomposites. Appl Phys Lett 2011; 98: 241112.

34 Khurgin JB, Sun G, Chen WT, Tsai WY, Tsai DP. Ultrafast thermal nonlinearity. Sci Rep 2015; 5: 17899.

35 Gaskell JM, Afzaal M, Sheel DW, Yates HM, Delfanazari K et al. Optimised atmospheric pressure CVD of monoclinic $\mathrm{VO}_{2}$ thin films with picosecond phase transition. Surf Coat Technol 2016; 287: 160-165.

36 Abb M, Wang YD, de Groot CH, Muskens OL. Hotspot-mediated ultrafast nonlinear control of multifrequency plasmonic nanoantennas. Nat Commun 2014; 5: 4869

37 Cueff S, Li DF, Zhou Y, Wong FJ, Kurvits JA et al. Dynamic control of light emission faster than the lifetime limit using $\mathrm{VO}_{2}$ phase-change. Nat Commun 2015; 6: 8636.

38 Chettiar UK, Engheta N. Modeling vanadium dioxide phase transition due to continuous-wave optical signals. Opt Express 2015; 23: 445-451.

39 Pashkin A, Kübler C, Ehrke H, Lopez R, Halabica A et al. Ultrafast insulator-metal phase transition in $\mathrm{VO}_{2}$ studied by multiterahertz spectroscopy. Phys Rev B 2011; 83: 195120.

40 Hilton DJ, Prasankumar RP, Fourmaux S, Cavalleri A, Brassard D et al. Enhanced photosusceptibility near $T_{c}$ for the light-induced insulator-to-metal phase transition in vanadium dioxide. Phys Rev Lett 2007; 99: 226401.

This work is licensed under a Creative Commons Attribution 4.0 International License. The images or other third party material in this article are included in the article's Creative Commons license, unless indicated otherwise in the credit line; if the material is not included under the Creative Commons license, users will need to obtain permission from the license holder to reproduce the material. To view a copy of this license, visit http://creativecommons.org/licenses/by/4.0/

(C) The Author(s) 2016

Supplementary Information for this article can be found on the Light: Science \& Applications' website (http://www.nature.com/lsa). 